\title{
Small-field perimetry applied to different types of retinitis pigmentosa: preliminary results
}

\author{
Alejandro H. Gloriani ${ }^{1}$, Isabel Arranz ${ }^{1}$, Beatriz M. Matesanz ${ }^{1}$, Luis A. Issolio ${ }^{2}$, José A. Menéndez ${ }^{3}$, Santiago Mar ${ }^{1}$, Alicia Galindo ${ }^{4}$ and Juan $^{*}$ \\ A. Aparicio ${ }^{1 *}$ \\ ${ }^{1}$ Departamento de Física Teórica, Atómica y Óptica, Universidad de Valladolid, Spain \\ ${ }^{2}$ Departamento de Luminotecnia, Luz y Visión, Universidad Nacional de Tucumán, e Instituto de Luz, Ambiente y Visión, CONICET-UNT, Argentina \\ ${ }^{3}$ Departamento de Estadística e Investigación Operativa, Universidad de Valladolid, Spain \\ ${ }^{4}$ Servicio de Oftalmología del Hospital Universitario Río Hortega, Valladolid, Spain
}

\begin{abstract}
Purpose: To measure the sensitivity of small retinal areas by reducing the influence of the adaptation state of the rest of the retina. To assess the functional behaviour of the retina of several patients affected by different types of retinitis pigmentosa (RP).

Methods: A prospective study was performed. Five eyes of patients with retinal diseases and different genetic mutations were studied at the University of Valladolid: three typical RP, one cone rod dystrophy (CRD) and one Bardet-Biedl syndrome (BBS). One of the RP patients was analysed again 18 months after the first measurements. A two-maxwellian view optical arrangement was used to measure the luminance thresholds of retinal areas 1 deg sized under dark and low photopic adaptation conditions. Linear regression fits of these thresholds vs. eccentricity were used to compare the patients' sensitivity with the control group.
\end{abstract}

Results: The slopes of the linear fits of the other typical RP patients were higher than those of the control group. The parameters of the linear fits of the RP patient measured twice changed significantly with time.

Conclusions: The designed psychophysical method provides, in all cases, coherent results with the expected ones according to the genetic mutation of the patients. The technique seems to be very sensitive to changes in the retinal response. It can be easily incorporated as a technical complement to the traditional perimetry.

\section{Introduction}

Retinitis pigmentosa is a vague term which embraces different retinal disorders featured by progressive visual disability, photoreceptor cell loss and finally, by retinal atrophy. Current data estimate that $\mathrm{RP}$ affects 1:4000 people worldwide [1,2]. RP is associated with an important clinical and genetic heterogeneity [3]. Although common clinical criteria exist to define it [4], the precise identification of the type of retinal disorder requires frequently the use of expensive techniques such as electroretinography (ERG). Genetic analysis is also a helpful and powerful tool but this is more expensive and results are obtained after a long time. Nevertheless, this analysis provide relevant information concerning the inheritance patterns in RP [5]. At the last stages of RP, there are different clinical presentations of the dystrophy which affect all retinal layers, making the precise identification difficult.

Psychophysical techniques have been used in the past to assess the retinal behaviour in RP patients [6-8]. When combined with electroretinography (ERG), they have allowed to correlate high cone or rod thresholds with retinal slimming [9]. In combination with the Optical Coherence Tomography (OCT), they have allowed to correlate the retinal sensitivity loss and photoreceptor impairment with the shortening of the photoreceptor inner-outer segment junction [10]. In the clinical practice, automated perimetry represents the most frequently employed psychophysical technique to measure the luminance thresholds and the useful visual field. However, there are important reasons which lead us to think that their potential capabilities may have not been completely exploited yet. First, in the traditional perimetry, the measured sensitivity is not only dependent on the local response of the retinal area where the probe is shown, but also with the adaptation luminance of the rest of the retina [11]. Since in RP patients the functionality of photoreceptors is altered across the whole retina, it looks convenient to measure sensitivity to light of each retinal area by minimizing the influence of the adaptation state of neighbour regions which are usually heterogeneously damaged. This can be achieved by reducing as much as possible the size of the adaptation field employed in the perimetry. Second, it looks convenient to perform measurement under conditions not dependent on the dilation or constriction properties of pupils' patient, which may be altered otherwise. Under these conditions, the obtained measurements would provide more feasible information about the local response of each part of the retina.

In this work, we have built a perimeter based on maxwellian illumination capable of measuring the retinal response of discrete and small retinal areas. The following study has been performed. Retinal sensitivity in the first $12^{\circ}$ temporal retina has been measured at scotopic and low photopic levels in subjects with different types of RP, all them

Correspondence to: Juan Antonio Aparicio. Departamento de Física Teórica, Atómica y Óptica. Universidad de Valladolid, Paseo de Belén 7, 47011 Valladolid, Spain, Tel: 34983423 267; Fax: 34983423 013; E-mail: juanantonio.aparicio@uva.es

Key words: cone-rod dystrophy, photoreceptors cells, psychophysics, retinitis pigmentosa Received: January 20, 2017; Accepted: February 07, 2017; Published: February 10,2017 
in their mid or end stage of their pathologies [2].

With this device, we have addressed the following questions: 1) is it possible to correlate sensitivity measurements with expected behaviours according to the genetic analysis in different types of RP?, 2) is it possible to determine functional trends with eccentricity?, 3) are these trends useful as indicators of the pathology evolution?

\section{Methods}

\section{Subjects}

The right eyes of five RP patients, as well as eight ocularly healthy subjects, with mean age of $34 \pm 9$ years, and $34 \pm 8$ years respectively, were included in the study. An ophthalmic assessment was performed on all participants including visual acuity (log MAR), colour vision (Farnsworth D-15), visual fields (Humphrey Field Analyzers, HFA; Carl Zeiss Meditec Inc., Dublin, CA) and fundus examination (Goldman lens). Additional exams such as optical coherence tomography, OCT, (Carl Zeiss Meditec, Dublin, California, USA), a fluorescein angiography, full field electroretinogram ffERG (ISCEV Standard) or multifocal-ERG allowed the diagnostics. The type of genetic mutation was determined in all of them (Table 1). An informed consent was obtained from all volunteers. The study was approved by the Institutional Ethical Committee. The tenets of the Declaration of Helsinki were followed. The only inclusion criterion required in this study was the ability to detect the fixation tests described below.

\section{Experimental arrangement}

A scheme of the set-up is shown in Figure 1. This has been described in detail previously [12]. A two-maxwellian view optical system was used. It incorporates two circular concentric beams (incandescent sources S1 and S2, colour temperature around $3000 \mathrm{~K}$ ): a probe beam (stimulus) and an adaptation beam, being their maximum attainable luminances around 6500 and $120 \mathrm{~cd} / \mathrm{m}^{2}$ respectively. Their angular sizes are $0.45^{\circ}$ and $1^{\circ}$ respectively, as seen from the observer's entrance pupil (E). Shutters Ob1 and Ob3 allow their time exposure control. Neutral density filters (F1-F6) permitted to change stimulus and adaptation field luminances in steps of $0.1 \log$ units. Two fixations tests built with ultraluminescent red LEDs are included: one for the foveal measurements (FMFT) and another for the peripheral ones (EMFT), whose luminances are adaptable to patients' visual capabilities. In order to avoid head movements, the observer bites on a bite bar made on a dental compound during measurements.

A CCD camera allows control of the subject's pupil alignment.

\section{Measurements performed}

Some previous trials led us to restrict our measurements to the temporal retina, from fovea $\left(0^{\circ}\right)$ to $12^{\circ}$ eccentricity in steps of $3^{\circ}$ and only to right eyes to make results fully comparable. Previously to measurements, all subjects adapted to darkness $\left(5 \times 10^{-6} \mathrm{~cd} / \mathrm{m}^{2}\right)$ during 30 minutes. They performed two series of measurements. In one of

Table 1. Clinical characteristics of patients $(\mathrm{AD}=$ autosomal dominant, $\mathrm{AR}=$ autosomal recessive $)$.

\begin{tabular}{|c|c|c|c|c|c|}
\hline Patient & Mode of inheritance//Type of RP & Gender & Age & Acuity (OD) & Genetic disorder \\
\hline TRP1 & AR / typical RP & F & 38 & 0.18 & 0.10 \\
\hline TRP2 & AD / typical RP & F & 33 & 0.40 & mutations inUSH2A gene \\
\hline TRP3 & AR / RCD & M & 25 & 43 & Mutation in RHO gene \\
\hline CRD & AD / CRD & M & 43 & Mution in CERKLgene \\
\hline BBS & AR / Bardet-Biedlsyndrome & M & 30 & 1.30 & Mutation in PRPH gene \\
\hline
\end{tabular}

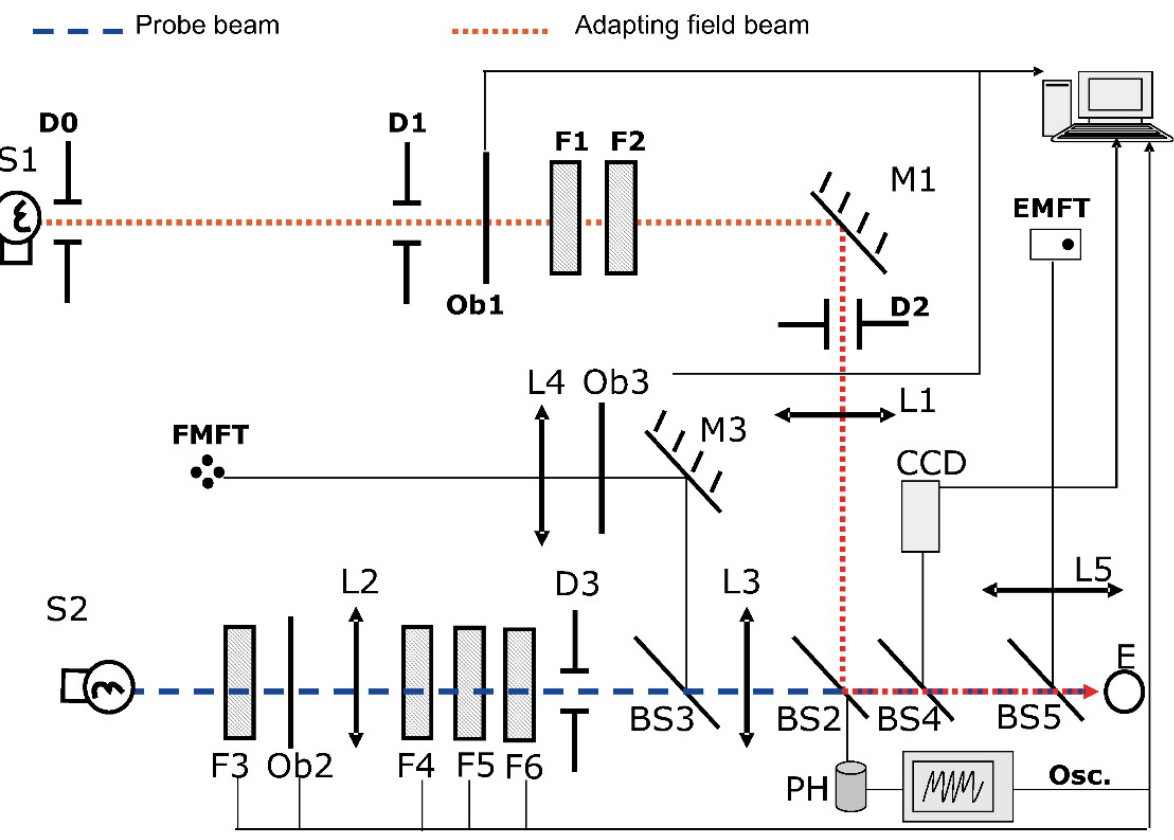

Figure 1. Experimental arrangement (reproduced with permission of the copyright owner). 
them, luminance detection thresholds were measured with shutter Ob1 closed under dark adaptation conditions (DA) [13]; in the other one and, after 10 minutes of exposure to a steady adaptation field of 5 $\mathrm{cd} / \mathrm{m}^{2}$, thresholds were determined under light adaptation conditions (LA). This luminance value is above the upper limit of mesopic range [14]. The stimulus duration was always $40 \mathrm{msec}$. Time between two consecutive presentations of stimuli was $10 \mathrm{sec}$ for the normal subjects, and $30 \mathrm{sec}$ for the RP patients, in order to avoid afterimages. The order of these series and the order in which eccentricities were analysed were always randomized. A natural pupil was employed, since its diameter was always greater than the size of the imaged filament on the entrance pupil plane. No drugs were provided to the participants in the study. All these measurements as well as the ophthalmologic exam were repeated for patient TRP2 18 months later in order to detect possible changes in the pathology evolution. In this case, the patient herself requested the study by arguing loss of vision particularly at dim lighting conditions.

\section{Statistical analysis}

Luminance thresholds were measured by employing the limits method. The log incremental luminance thresholds $\log L t\left(L t\right.$ in $\left.\mathrm{cd} / \mathrm{m}^{2}\right)$ vs. eccentricity $e$ (in degrees) were fitted to linear functions $\log L t=a+b e$ at both DA and LA conditions, for the average thresholds measured on the control group, and individually for each patient. The standard errors $\sigma a$ and $\sigma b$ were calculated for each fit. Linear fits have been preferred to higher-order polynomial fits since they provide significant information with a simpler analysis.

\section{Results}

In Figure 2 thresholds $\log L t^{\mathrm{DA}}$, measured in scotopic conditions (DA) are depicted vs. eccentricity. In each panel we have included the data for each individual, the fit to his/her data, the average of thresholds for the control group with their $95 \%$ confidence interval, and its linear fit. Intercept data $a \pm \sigma a$ and the slope $b \pm \sigma b$ are shown in the third column of Table 2 .

As seen in Figure 2, thresholds for normal subjects decrease from fovea to $6^{\circ}$ eccentricity and then slightly increase up to $12^{\circ}$ peripheral retina, being the confidence intervals very small in all cases. Results in Table 2 show that, on average, the slope of the fit to these data is statistically negative. Concerning the results for our five patients, the slope of the fits for TRP1 and TRP2 is positive, being the intercept (ordinate at null eccentricity) significantly greater than for the control

Table 2. Intercepts (a), slopes (b) and their standard errors obtained from the linear fits to theexperimental data of each individual and for the control group. Data for DA and LA conditions are included.

\begin{tabular}{|c|c|c|c|}
\hline & & DA & LA \\
\hline Control & $\mathrm{a} \pm \sigma \mathrm{a}$ & $-3.49 \pm 0.51$ & $0.51-0.25 \pm 0.31$ \\
\hline & $b \pm \sigma b$ & $-0.07 \pm 0.03$ & $0.11 \pm 0.02$ \\
\hline TRP1 & $a \pm \sigma a$ & $-2.19 \pm 0.74$ & $-0.66 \pm 0.39$ \\
\hline TRP2 2012 & $b \pm \sigma b$ & $0.21 \pm 0.10$ & $0.26 \pm 0.05$ \\
\hline TRP2 2013 & $a \pm \sigma a$ & $-2.89 \pm 0.61$ & $-0.25 \pm 0.12$ \\
\hline & $b \pm \sigma b$ & $0.32 \pm 0.07$ & $0.17 \pm 0.02$ \\
\hline TRP3 & $a \pm \sigma a$ & $-2.82 \pm 0.57$ & $-0.04 \pm 0.11$ \\
\hline & $b \pm \sigma b$ & $0.41 \pm 0.07$ & $0.20 \pm 0.02$ \\
\hline CRD & $a \pm \sigma a$ & $0.51 \pm 0.87$ & $1.10 \pm 0.55$ \\
\hline & $b \pm \sigma b$ & $-0.12 \pm 0.11$ & $0.11 \pm 0.07$ \\
\hline BBS & $a \pm \sigma a$ & $-1.93 \pm 0.86$ & $0.44 \pm 0.18$ \\
\hline & $b \pm \sigma b$ & $-0.02 \pm 0.11$ & $0.10 \pm 0.02$ \\
\hline & $a \pm \sigma a$ & $0.71 \pm 0.18$ & $1.95 \pm 0.15$ \\
\hline & $b \pm \sigma b$ & $0.22 \pm 0.02$ & $0.14 \pm 0.03$ \\
\hline
\end{tabular}
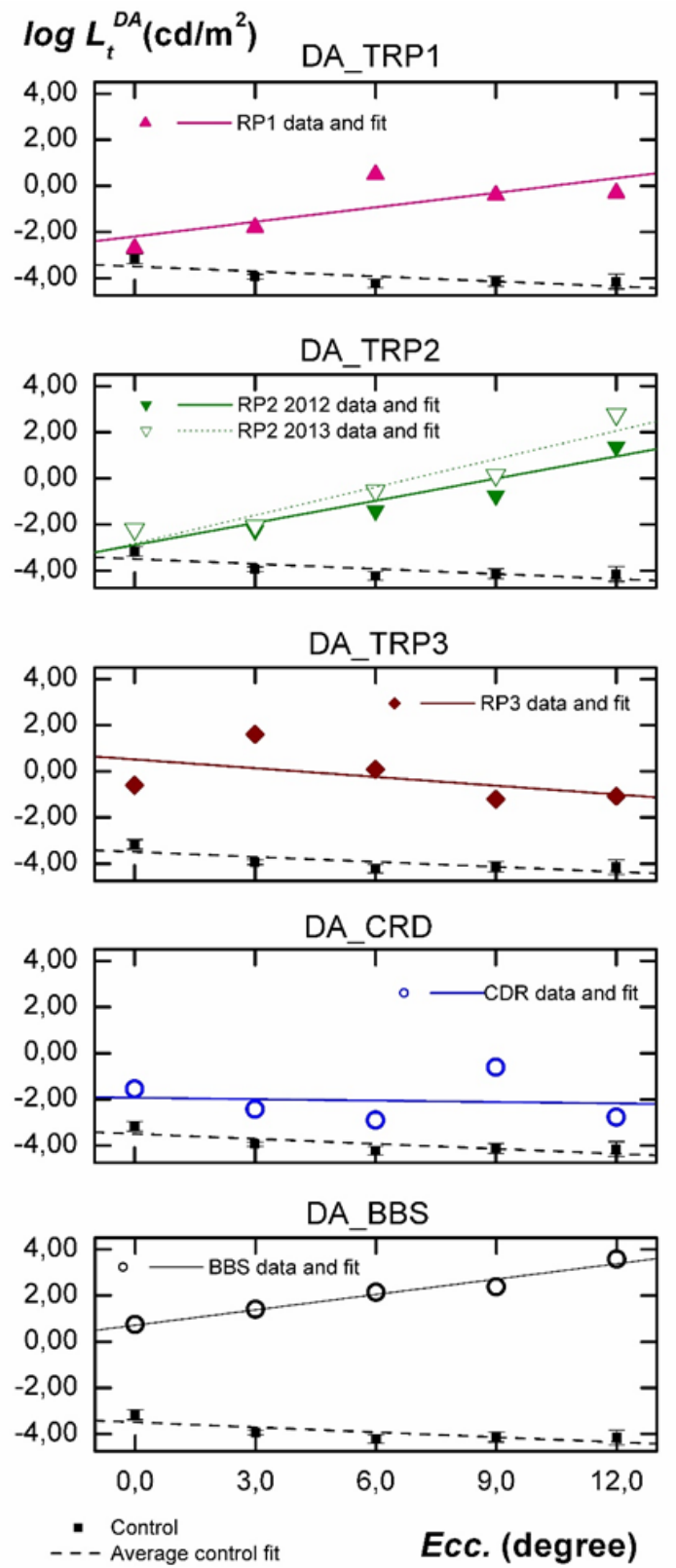

Figure 2. Analysis of the influence of eccentricity on the absolute thresholds for each individual at DA conditions. Subjects are labelled as indicated in Table 1. The linear fit for each subject (solid line), the average of thresholds for the control group (solid squares) with their $95 \%$ confidence interval, and the linear fit to their data (dashed line) are included.

group (1.3 log units for RP1, 0.60 log units for RP2 in 2012 and 0.67 for this patient in 2013). As can also be seen in Figure 2 and in Table 2, the functional behaviour of thresholds with eccentricity for TRP3 is more similar to that observed for CRD. In both cases, the sign of the slope is negative being also the intercept significantly greater than for the control group (4.0 log units for TRP3 and $1.56 \log$ units for CRD) For the BBS patient, the obtained intercept is $4.2 \log$ units higher than for the control group and the slope is statistically equal to those obtained for TRP1 and TRP2.

In Figure 3 luminance thresholds $\log L t^{L A}$, measured under adaptation to a $5 \mathrm{~cd} / \mathrm{m}^{2}$ steady and low photopic field (LA) are plotted vs. eccentricity. The order of individuals, symbols, and labels are identical to those employed in Figure 2. The parameters $a \pm \sigma a$ and $b \pm \sigma b$ 

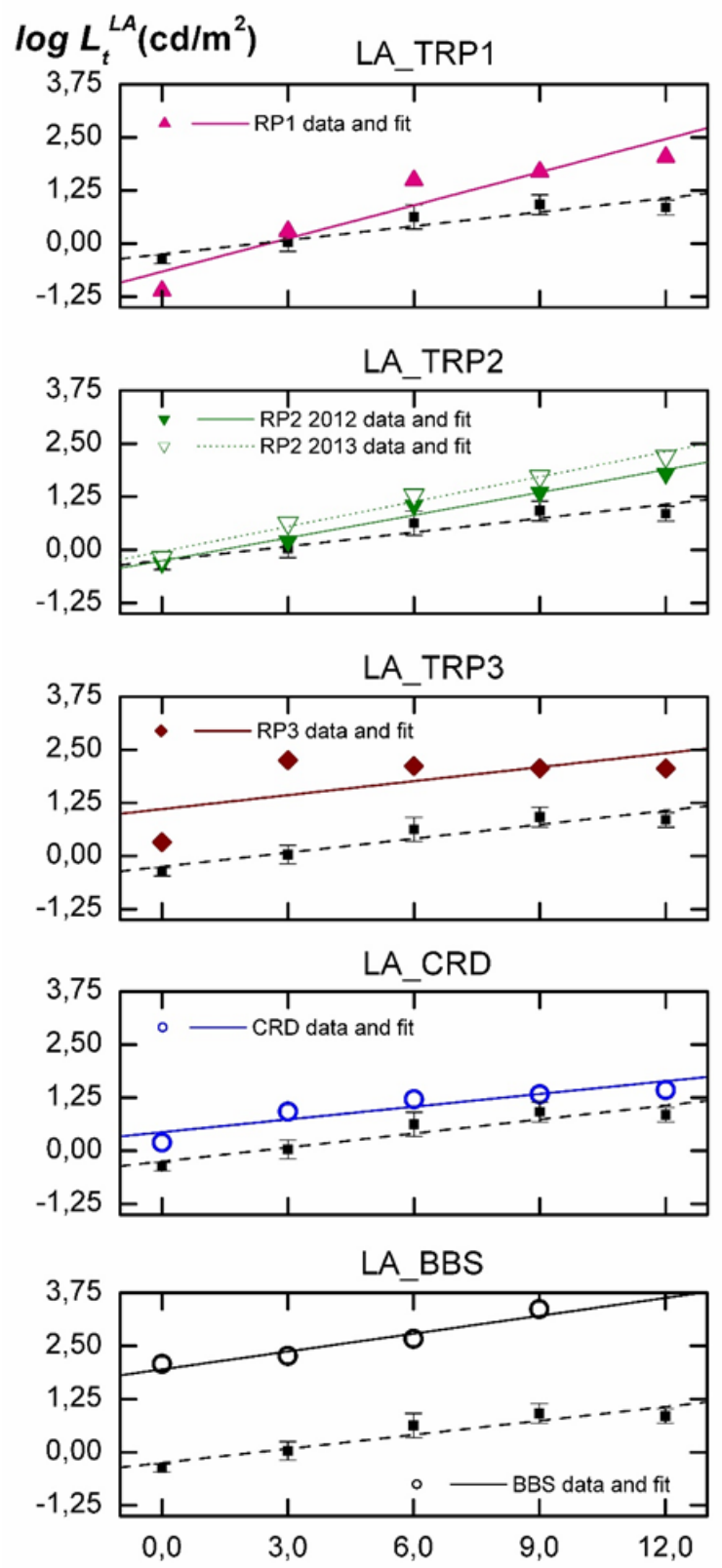

- Control

- - Average control fit

Ecc. (degree)

Figure 3. Individual analysis of the influence of eccentricity on luminance thresholds measured under adaptation to a steady field of $5 \mathrm{~cd} / \mathrm{m}^{2}$. Comparison with results obtained for the control group.

for each fit are shown in the fourth column of Table 2.

As seen in Figure 3, luminance thresholds for the control group under LA conditions increase from fovea to $9^{\circ}$ and then tend to remain stable, being the slope of the fit positive. The slopes of the fits for TRP 1 and TRP2 patients are also positive and higher than for normal individuals (as at DA). However, the intercepts are not statistically different. For CRD and TRP3 patients, slopes are not statistically different from one another and, as was the case at DA, are statistically equal to the one obtained for the control group. However, the intercepts are greater for CRD (0.69 log units) and TRP3 patient (1.35 log units) than for normal subjects. With regard to the BBS patient, measurements under
LA show a behavior which is more similar to CRD or TRP3 than to TRP1 or TRP2, contrary to the one observed under DA. Compared to the control group, the intercept of the fit is $2.2 \log$ units greater, but its slope is not statistically different.

Comparisons between results obtained in the two series of measurements performed on patient TRP2 yield a significant increase in the ordinates of the fits at LA conditions and a statistically significant increase in the slopes, particularly at DA conditions.

\section{Discussion}

The procedure employed has quantified precisely the retinal sensitivity in DA and LA conditions with high spatial resolution, by minimizing the effects of pupil diameter and the response of other retinal areas. The statistical analysis based on linear fits offer some interesting conclusions.

First, with the exception of fovea, the results in Figure 2 for the control group are linked to the rod response. The negative slope in the fit is explained by the increasing rod density with eccentricity and the amacrine AII cell concentration around $6^{\circ}$ [15]. If we consider the genetic mutations of our TRP1 and TRP2 patients, it is reasonable to expect an increase of threshold luminances with eccentricity at DA [2], being the measured luminance thresholds mostly due to the cone response. This is exactly what is observed for these two patients in Figure 2. The severe rod disability can be inferred from the slope of the fit, which is positive for them, as well as for the great differences in luminance threshold relative to normal subjects. It is remarkable that the functional behaviour for TRP3 is opposite to that observed for TRP1 and TRP2. However, the measured intercept is very high and the slope is negative for this patient.

This corresponds to a more severely damaged central retina and a better functionality at peripheral regions. The different behaviour observed in TRP3, more similar to CRD, can be explained again from the type of genetic mutation causing this retinal pathology. Mutation on CERKL gen produces a retinal disorder featured by a rapid and progressive decline of visual function [16], reducing particularly the central field of view and the visual acuity. As described in the literature and shown in Figure 2, these are also typical manifestations observed in CRD patients, which may lead to wrong diagnostics [17].

Second, when analyzing Figure 3, we must consider that at $5 \mathrm{~cd} /$ $\mathrm{m}^{2}$, sensitivity in normal subjects is mostly but not exclusively due to cones [14]. However, due to the severe rod disability shown in Figure 2 for patients TRP1 and TRP2, the observed increasing differences in thresholds relative to normal subjects (Figure 3) may indicate some damage on their peripheral cones. These differences are enough to be detected by mean of the statistically significant increment observed in the slope of the fits. This increasing disability of cones with eccentricity is well known in typical RP and has been recently described as a result of a neural reorder occurring in the postreceptoral pathways after the local retinal dysfunction [18-21]. It is interesting to remark that, as happened with rod response in Figure 2, in Figure 3 slopes of linear fits for TRP3 and CRD patients are very similar to those measured for the control group. Unlike TRP1 or TRP2, the genetic mutations observed in our CRD and TRP3 patients seem to produce a functional decay which is very similar for the first $12^{\circ}$ of eccentricity. It is also remarkable that all these conclusions are obtained in spite of the local damages existing in the retina of our patients, many of them not being detected by the automated perimetry. The ability to detect these 
damages and the stability of the obtained results support the strength and sensitivity of our device.

Third, as can be inferred from above discussion, the slope of the performed fits gives us significant information of the progression of cellular damage with eccentricity while the increase of the intercept relative to its value for the control group informs us about how the cellular damage reached the central retina. Furthermore, when we extend this analysis to the two sets of measurements performed to subject TRP2, a new relevant result arises. The statistically significant increase observed in the linear fits for TRP2 18 months after the first measurements informs us about the speed of her pathology progression with time. This progression is faster for rods (more increment in the slope) than for cones.

Finally, results of the BBS individual deserve specific comments. As can be seen from his luminance thresholds in Figure 2 and 3, the pathology in this patient was at its very final stage. In fact, conventional perimetry is not applicable for him, due to the inability to see the fixation point or to detect the stimulus due to their insufficient luminances With our experimental arrangement, the high attainable luminance in the stimulus $\left(6500 \mathrm{~cd} / \mathrm{m}^{2}\right)$ has allowed sensitivity measurements at all proposed retinal locations, even though some of them were considered as blindness zone according to the automated static perimetry. However, this luminance has not been enough to measure the BBS thresholds at $12^{\circ}$ eccentricity under LA. As shown in Figure 2, the great differences in threshold luminance and the change of the slope relative to the control group inform us about the absence of any rod sensitivity (confirmed by full field ERG). The similar slope observed in Figure 3 relative to the control group informs us about a very sever cellular damage similarly extended all along the considered retinal area.

As a conclusion, the preliminary results shown in this work prove that the analysis of thresholds vs eccentricity by linear regressions, can be used as a complementary technique in the clinical practice. They are fully correlated with the expected retinal behaviour according to the genetic mutations and provide significant information about the retinal rod and cone functionality. Finally, this technique has not only proven its usefulness to assess the evolution of these pathologies with eccentricity and time, but also can be used as a screening of retinal disorders in very initial stages. In these cases, where a significant rod functionality may exist, the luminance employed to analyze cone response in LA measurements should be increased at values around $100 \mathrm{~cd} / \mathrm{m}^{2}$, where rods are fully saturated.

\section{Authorship and contributorship}

All authors satisfy the criteria of authorship and contributorship stated by the journal.

\section{Acknowledgments}

The authors thank Santiago González for his collaboration in the experimental arrangement as well as all the participants in the study. Juan A. Aparicio wants to thank the Organizacion Nacional de Ciegos de España for their help.

\section{Funding information}

Finance by the Ministerio de Economía y Competitividad under contract FIS2016-78037-P.

\section{Competing interest}

There is not conflict of interest for any author.

\section{References}

1. Haim M (2002) Epidemiology of retinitis pigmentosa in Denmark. Acta Ophthalmol Scand Suppl: 1-34. [Crossref]

2. Hamel C (2006) Retinitis pigmentosa. Orphanet J Rare Dis 1: 40. [Crossref]

3. Ayuso C, Millan JM (2010) Retinitis pigmentosa and allied conditions today: a paradigm of translational research. Genome Med 2: 34. [Crossref]

4. [No authors listed] (1983) Retinitis pigmentosa. A symposium on terminology and methods of examination. Ophthalmology 90: 126-131. [Crossref]

5. Cotta de Queiroz AC, Frasson M, dos Reis Veloso, CE, Arantes RR, Nehemy MB (2013) Clinical study and pattern of inheritance in patients with retinitis pigmentosa. Rev Bras Oftalmol 72: 26-28.

6. Greenstein VC, Hood DC, Siegel IM, Carr RE (1984) Retinitis pigmentosa: a psychophysical test of explanations for early foveal sensitivity loss. Invest Ophthalmol Vis Sci 25: 118-120. [Crossref]

7. Dagnelie G, Massof RW (1993) Foveal cone involvement in retinitis pigmentosa progression assessed through flash-on-flash parameters. Invest Ophth Vis Sci 34: 231242 .

8. Holopigian K, Seiple W, Greenstein VC, Hood DC, Carr RE (2001) Local cone and rod system function in patients with retinitis pigmentosa. Invest Ophthalmol Vis Sci 42: 779-788. [Crossref]

9. Apushkin MA, Fishman GA, Alexander KR, Shahidi M (2007) Retinal thickness and visual thresholds measured in patients with retinitis pigmentosa. Retina 27: 349-357. [Crossref]

10. Mitamura Y, Aizawa S, Baba T, Hagiwara A, Yamamoto S (2009) Correlation between retinal sensitivity and photoreceptor inner/outer segment junction in patients with retinitis pigmentosa. Br J Ophthalmol 93: 126-127. [Crossref]

11. Hayhoe MM (1990) Spatial interactions and models of adaptation. Vision Res 30: $957-$ 965. [Crossref]

12. Matesanz BM, Issolio L, Arranz I, de la Rosa C, Menéndez JA, et al. (2011) Temporal retinal sensitivity in mesopic adaptation. Ophthalmic Physiol Opt 31: 615-624. [Crossref]

13. Jacobson SG, Voigt WJ, Parel J-M, Apathy PP, Nghiem-Phu L, Myers SW et al (1986) Automated light-adapted and dark-adapted perimetry for evaluating retinitis pigmentosa. Ophthalmology 93: 1604-1611.

14. TC-1.4 (1978) Light as a true visual quantity: Principles of measurement. Paris: CIE Technical Report No. 41.

15. Mills SL, Massey SC (1999) AII amacrine cells limit scotopic acuity in central macaque retina: a confocal analysis of calretinin labeling. J Comp Neurol 411: 19-34. [Crossref]

16. Avila-Fernandez A, Riveiro-Alvarez R, Vallespin E, Wilke R, Tapias I, et al. (2008) CERKL mutations and associated phenotypes in seven Spanish families with autosomal recessive retinitis pigmentosa. Invest Ophthalmol Vis Sci 49: 2709-2713. [Crossref]

17. Hamel CP (2007) Cone rod dystrophies. Orphanet J Rare Dis 2: 7. [Crossref]

18. Scholl HPN, Kremers J (2000) Large phase differences between L-cone- and M-conedriven electroretinograms in retinitis pigmentosa. Invest Ophth Vis Sci 41: 3225-3233. [Crossref]

19. Scholl HP, Kremers J (2003) Alterations of L- and M-cone driven ERGs in cone and cone-rod dystrophies. Vision Res 43: 2333-2344. [Crossref]

20. Holopigian K, Shuwairi SM, Greenstein VC, Winn BJ, Zhang X, et al. (2005) Multifocal visual evoked potentials to cone specific stimuli in patients with retinitis pigmentosa. Vision Res 45: 3244-3252. [Crossref]

21. Parisi V, Ziccardi L, Stifano G, Montrone L, Gallinaro G, et al. (2010) Impact of regional retinal responses on cortical visually evoked responses: multifocal ERGs and VEPs in the retinitis pigmentosa model. Cli Neurophysiol 121: 380-385. [Crossref]

Copyright: (C)2017 Gloriani AH. This is an open-access article distributed under the terms of the Creative Commons Attribution License, which permits unrestricted use, distribution, and reproduction in any medium, provided the original author and source are credited. 